\title{
COMPACT ENDOMORPHISMS OF BANACH ALGEBRAS II
}

\author{
HERBERT KAMOWITZ, STEPHEN SCHEINBERG AND DENNIS WORTMAN
}

(Communicated by John B. Conway)

\begin{abstract}
In this note we exhibit examples which show that several conjectures concerning compact endomorphisms of commutative semisimple Banach algebras prove to be false.
\end{abstract}

In this sequel to an earlier paper [4], we answer several open questions about compact endomorphisms of commutative semi-simple Banach algebras. In so doing, we show that their behavior is quite diverse. We recall that a compact endomorphism of a Banach algebra $B$ is a compact linear map of $B \rightarrow B$ which preserves multiplication. Further, if $T$ is a non-zero endomorphism of $B$, and $X$ is the maximal ideal space of $B$, then there exists a map $\phi: X \rightarrow X$ such that $\widehat{T f}(x)=\hat{f}(\phi(x))$ for all $f \in B, x \in X$. We will denote the $n$th iterate of $\phi$ by $\phi_{n}$.

The main theorem in [4] states that if $T$ is a non-zero compact endomorphism of a commutative semi-simple Banach algebra $B$ with maximal ideal space $X$, and if $\widehat{T f}=\hat{f} \circ \phi$, then $\bigcap \phi_{n}(X)$ is finite. As a consequence, if $X$ is connected and $B$ has an identity then $\cap \phi_{n}(X)$ is a singleton, while if $B$ has no identity then 0 is the only compact endomorphism.

Clearly, if $B$ and $X$ are as described above, and if $B$ has an identity 1 , then $T: f \rightarrow \hat{f}\left(x_{0}\right) 1$ is a compact endomorphism for each $x_{0} \in X$. Much of what follows is concerned with the question of when such endomorphisms are the only non-zero compact endomorphisms.

Two natural examples are $C(X)$ for compact connected $X$ and the disc algebra $A$. For the former, every non-zero compact endomorphism has the form $T: f \rightarrow \hat{f}\left(x_{0}\right) 1$ for some $x_{0} \in X$, while for the disc algebra $A$, if $T f=f \circ \phi$, then $T$ is compact if, and only if, either $\phi(z)=$ constant for all $z,|z| \leq 1$, or else, for some $\delta>0,|\phi(z)| \leq 1-\delta$ for all $z,|z| \leq 1$ [3]. In the case of the disc algebra, the unit circle $\Gamma$ is both the Silov boundary and the strong boundary, and thus $T: f \rightarrow f \circ \phi$ is a compact endomorphism of $A$ if, and only if, $\phi$ is constant or $\Gamma \cap($ range $\phi)=\varnothing$.

Received by the editors October 18, 1988 and, in revised form, February 16, 1989.

1980 Mathematics Subject Classification (1985 Revision). Primary 47B38, 46J99, 47B05. 
Attempts to generalize the last statement fail. Again, with the same meanings for $X, T, \phi$, there are examples of uniform algebras on $X$ where (i) $T$ is compact and $\phi(X)$ intersects the Silov boundary, yet $\phi$ is not constant, and where (ii) $\phi(X)$ does not intersect the Silov boundary, but $T$ is not compact. Also, $\bigcap \phi_{n}(X)$ can be singleton, and still $T$ is not compact [4].

On a positive note, we list the following results. The first follows from the fact that if $T: B \rightarrow B$ is a compact endomorphism of the commutative semisimple Banach algebra $B$, then the enducing map $\phi$ is continuous in the norm topology of $B^{*}$. More general results along these lines may be found in [7].

Theorem A. Suppose $B$ is a uniform algebra on a compact connected space $X$ such that every point in $X$ is a one point Gleason part. If $T$ is a non-zero compact endomorphism of $B$, then $T f=f\left(x_{0}\right) 1$ for some $x_{0} \in X$.

Theorem B [4, p. 322]. If $B$ is a uniform algebra on a compact connected space $X$ and $T$ is a non-zero compact endomorphism of $B$ with $T f=f \circ \phi$, and if for some $x_{0} \in X, \phi\left(x_{0}\right)$ belongs to the strong boundary of $B$, then $T f=f\left(\phi\left(x_{0}\right)\right) 1$.

Theorem C [5]. If $B$ is the Banach algebra generated by a hermitian element $g$ with connected spectrum (say, $\operatorname{spec} g=[a, b]$ ), then every non-zero compact endomorphism $T$ on $B$ has the form $T f=\hat{f}\left(x_{0}\right) 1$ for some $x_{0} \in[a, b]$.

We remark, too, that if $I=[0,1]$ and $B=C^{(n)}\left(I^{m}\right)$, it can be shown that every non-zero compact endomorphism of $B$ has the form $T f=f\left(\mathbf{x}_{0}\right) 1$ for some $\mathbf{x}_{0} \in I^{m}$. Further, for the algebras, $\operatorname{Lip}_{\alpha}[0,1], 0<\alpha \leq 1$, and $\operatorname{lip}_{\alpha}[0,1]$, $0<\alpha<1$, (see [1] for definitions) every non-zero compact endomorphism $T$ has the form $T f(x)=f\left(x_{0}\right) 1$, for some $x_{0} \in[0,1]$.

We observe that in all of these examples, whenever $B$ is a regular Banach algebra in the sense of Silov, each non-zero compact endomorphism $T$ has the form $T: f \rightarrow \hat{f}\left(x_{0}\right) 1$, for some $x_{0}$ in the maximal ideal space of $B$. This led to the conjecture that this fact holds in general. As the main result in this note, we shall demonstrate that this conjecture is false.

Before showing this, however, we remark that there exists a non-regular semisimple Banach algebra with connected maximal ideal space $X$ for which every non-zero compact endomorphism has the form $T: f \rightarrow \hat{f}\left(x_{0}\right) 1$, for some $x_{0} \in$ $X$. For such an algebra, we follow a suggestion of B. Cole and J. Wermer and utilize a construction due to Cole [8, Section 19]. Begin with the disk algebra $A$, whose maximal ideal space is $\Delta=\{z|| z \mid \leq 1\}$, and obtain the associated universal root algebra $A_{\Omega}$, with maximal ideal space $\Delta_{\Omega}$, which has these properties: there is a continuous map $\pi$ of $\Delta_{\Omega}$ onto $\Delta$, there is a projection $S$ of $C(\Delta)$ onto $\pi^{*} C(\Delta)=\{f \circ \pi \mid f \in C(\Delta)\}$ such that $S\left(A_{\Omega}\right)=\pi^{*} A$ and if $f$ is constant on $\pi^{-1}(z)$ then $S f$ has the same constant value on $\pi^{-1}(z)$, and for every $f \in A_{\Omega}$ there is a $g \in A_{\Omega}$ with $g^{2}=f$. As noted in [8], an immediate consequence of this last statement is that every Gleason 
part for $A_{\Omega}$ consists of a single point. From this and Theorem A, it follows that $A_{\Omega}$ admits no nontrivial compact endomorphisms. Further, it is easy to see from the construction that $\Delta_{\Omega}$ is connected. Finally, to see that $A_{\Omega}$ is not regular, suppose that it were. Then $A_{\Omega}$ is normal [8, Section 27]. Put $X=\pi^{-1}(\Gamma)=\pi^{-1}\{z|| z \mid=1\}$ and $Y=\pi^{-1}(0)$. If $f \in A_{\Omega}$ were 0 on $X$ and 1 on $Y$, there would exist $g \in A$ with $S f=g \circ \pi$, and it would follow that $g=0$ on $\Gamma$ and $g(0)=1$, an impossibility.

Finally, we present the following example.

Example of a regular, commutative, semi-simple Banach algebra which admits a non-trivial compact endomorphism. Let

$$
B=\left\{f \in C^{\infty}[0,1] \mid\|f\|=\sum_{n=0}^{\infty} \frac{\left\|f^{(n)}\right\|_{\infty}}{(n !)^{2}}<\infty\right\} .
$$

With the indicated norm and with pointwise addition and multiplication, $B$ is a regular commutative semi-simple Banach algebra, with maximal ideal space $[0,1]([2],[6])$. The required non-trivial compact endomorphism will be the map $T: f \rightarrow f(x / 2)$ on $B$. Clearly, $T$ is a linear multiplicative map of $B$ into $B$ which is (necessarily) bounded. To show that $T$ is a compact operator, let $f_{m} \in B$ with $\left\|f_{m}\right\|_{B} \leq 1$. The first step will be to show that there exists an element $G \in C^{\infty}[0,1]$ and a subsequence $\left\{f_{m_{j}}\right\}$ with $f_{m_{j}}^{(k)} \rightarrow G^{(k)}$ uniformly on $[0,1]$ for each $k \geq 0$. Since $\left\|f_{m}^{(k)}\right\|_{\infty} \leq(k !)^{2}$ for each $k$, it will follow that $\left\|G^{(k)}\right\|_{\infty} \leq(k !)^{2}$.

The subsequence is constructed as follows: the set $\left\{f_{m}^{\prime}\right\}$ is bounded in $C[0,1]$. Since the map $V: h \rightarrow \int_{0}^{x} h$ is a compact operator on $C[0,1]$, there exists a $g \in C[0,1]$ and a subsequence $\left\{f_{1, m}^{\prime}\right\}_{m=1}^{\infty}$ of $\left\{f_{m}^{\prime}\right\}$ with $\int_{0}^{x} f_{1, m}^{\prime} \rightarrow$ $g(x)$ uniformly on $[0,1]$. Consequently, $f_{1, m}(x)-f_{1, m}(0) \rightarrow g(x)$ uniformly on $[0,1]$. Then using a subsequence if necessary, we may assume that there exists an $a \in C$ with

$$
f_{1, m}(x) \rightarrow a+g(x)
$$

uniformly on $[0,1]$. Similarly, we can find $g_{1} \in C[0,1]$ and a subsequence $\left\{f_{2, m}\right\}_{m=1}^{\infty}$ of $\left\{f_{1, m}\right\}_{m=1}^{\infty}$ with $\int_{0}^{x} f_{2, m}^{\prime \prime} \rightarrow g_{1}(x)$ uniformly on [0,1]. Again using a subsequence if necessary, we may assume the existence of an $a_{1} \in C$ such that

$$
f_{2, m}^{\prime}(x) \rightarrow a_{1}+g_{1}(x)
$$

uniformly on $[0,1]$. Since $\left\{f_{2, m}\right\}$ is a subsequence of $\left\{f_{1, m}\right\}$, we have from $(1)$,

$$
f_{2, m}(x) \rightarrow a+g(x)
$$

uniformly on $[0,1]$. Let $G(x)=a+g(x)$. Then $G \in C[0,1]$, and

$$
f_{2, m}(x) \rightarrow G(x)
$$


uniformly on $[0,1]$. From (2), we have by integration

$$
f_{2, m}(x)-f_{2, m}(0) \rightarrow a_{1} x+\int_{0}^{x} g_{1}
$$

and from (4), we find

$$
f_{2, m}(x)-f_{2, m}(0) \rightarrow G(x)-G(0)=G(x)-a .
$$

Thus,

$$
G(x)=a+a_{1} x+\int_{0}^{x} g_{1},
$$

so $G$ is differentiable on $[0,1]$ and $G^{\prime}(x)=a_{1}+g_{1}(x)$. Thus, $G \in C^{1}[0,1]$, and $f_{2, m}^{\prime}(x) \rightarrow G^{\prime}(x)$ uniformly on $[0,1]$. Applying the above argument inductively, we find that $G \in C^{\infty}[0,1]$, and we obtain a nested sequence $\left\{f_{k, m}\right\}$ of subsequences of $\left\{f_{m}\right\}$ having the property that

$$
f_{k, m}^{(k-1)}(x) \rightarrow G^{(k-1)}(x) \quad \text { as } m \rightarrow \infty
$$

uniformly on $[0,1]$ for each $k$. We note that $G$ need not be in $B$.

Letting $f_{m_{j}}=f_{j+1, j}$ we have the following:

(i) $\left\|f_{m_{j}}^{(k)}\right\|_{\infty} \leq(k !)^{2}$ for each $k$.

(ii) $f_{m_{j}}^{(k)}(x) \rightarrow G^{(k)}(x)$ uniformly in $x$ on $[0,1]$, for each $k$.

(iii) $\left\|G^{(k)}\right\|_{\infty} \leq(k !)^{2}$ for each $k$.

In order to show that $T$ is compact, it suffices to show that $\left\{T f_{m_{j}}\right\}$ converges in $B$. We remark that for $f \in B,(T f)^{(k)}(x)=f^{(k)}(x / 2) / 2^{k}$.

Let $\Gamma(x)=G(x / 2)$. We will show that $\Gamma \in B$ and that $T f_{m_{j}} \rightarrow \Gamma$ in the norm of $B$. (Note that $\Gamma^{(k)}(x)=G^{(k)}(x / 2) / 2^{k}$.) Indeed,

$$
\sum_{k=0}^{\infty} \frac{\left\|\Gamma^{(k)}\right\|_{\infty}}{(k !)^{2}}=\sum_{k=0}^{\infty} \frac{\left\|G^{(k)}(x / 2)\right\|_{\infty}}{2^{k}(k !)^{2}} \leq \sum_{k=0}^{\infty} \frac{(k !)^{2}}{2^{k}(k !)^{2}}=\sum_{k=0}^{\infty} \frac{1}{2^{k}}=2 .
$$

Therefore, $\Gamma \in B$. Further,

$$
\begin{aligned}
\sum_{k=0}^{\infty} \frac{\left\|\left(T f_{m_{j}}\right)^{(k)}-\Gamma^{(k)}\right\|_{\infty}}{(k !)^{2}}= & \sum_{k=0}^{\infty} \frac{\left\|\frac{1}{2^{k}} f_{m_{j}}^{(k)}\left(\frac{x}{2}\right)-\frac{1}{2^{k}} G^{(k)}\left(\frac{x}{2}\right)\right\|_{\infty}}{(k !)^{2}} \\
= & \sum_{k=0}^{N} \frac{\left\|f_{m_{j}}^{(k)}\left(\frac{x}{2}\right)-G^{(k)}\left(\frac{x}{2}\right)\right\|_{\infty}}{2^{k}(k !)^{2}} \\
& +\sum_{k=N+1}^{\infty} \frac{\left\|f_{m_{j}}^{(k)}\left(\frac{x}{2}\right)-G^{(k)}\left(\frac{x}{2}\right)\right\|_{\infty}}{2^{k}(k !)^{2}} .
\end{aligned}
$$


Since $\left\|f_{m_{j}}^{(k)}(x / 2)-G^{(k)}(x / 2)\right\|_{\infty} \leq 2(k !)^{2}$,

$$
\sum_{k=N+1}^{\infty} \frac{\left\|f_{m_{j}}^{(k)}\left(\frac{x}{2}\right)-G^{(k)}\left(\frac{x}{2}\right)\right\|_{\infty}}{2^{k}(k !)^{2}} \leq \sum_{k=N+1}^{\infty} \frac{1}{2^{k-1}}=\frac{1}{2^{N-1}}
$$

Therefore,

$$
\sum_{k=0}^{\infty} \frac{\left\|\left(T f_{m_{j}}\right)^{(k)}-\Gamma^{(k)}\right\|_{\infty}}{(k !)^{2}} \leq \sum_{k=0}^{N} \frac{\left\|f_{m_{j}}^{(k)}\left(\frac{x}{2}\right)-G^{(k)}\left(\frac{x}{2}\right)\right\|_{\infty}}{2^{k}(k !)^{2}}+\frac{1}{2^{N-1}}
$$

This implies that

$$
\underset{j \rightarrow \infty}{\limsup }\left\|T f_{m_{j}}-\Gamma\right\| \leq \frac{1}{2^{N-1}} \text { for all } N,
$$

whence $T f_{m_{j}} \rightarrow \Gamma$ in the norm of $B$. Hence $T$ is compact on $B$ and the example is complete.

Essentially the same argument applies to maps $f(x) \rightarrow f(c x)$ and $f(x) \rightarrow$ $f(c(1-x)), 0<c<1$. An interesting problem is to characterize all compact endomorphisms of algebras of this type.

\section{REFERENCES}

1. W. G. Bade, B. C. Curtis Jr., and H. G. Dales, Amenability and weak amenability for Beurling and Lipschitz algebras, Proc. Lond. Math. Soc. (3) 55 (1987), 359-377.

2. H. G. Dales and A. M. Davie, Quasianalytic Banach function algebras, J. Funct. Anal. 13 (1973), 28-50.

3. H. Kamowitz, Compact operators of the form $u C_{\varphi}$, Pacific J. Math. 80 (1979), 205-211.

4. __ Compact endomorphisms of Banach algebras, Pacific J. Math. 89 (1980), 313-325.

5. H. Kamowitz and D. Wortman, Endomorphisms of an extremal algebra, Proc. Amer. Math. Soc. 104 (1988), 852-858.

6. A. G. O'Farrell, Polynomial approximation of smooth functions, J. Lond. Math. Soc. (2) 28 (1983), 496-596.

7. S. Ohno and J. Wada, Compact endomorphisms on function algebras, Tokyo J. Math. 4 (1981), 105-112.

8. E. L. Stout, The theory of uniform algebras, Bogden and Quigley, Inc., New York, 1971.

(H. Kamowitz and D. Wortman): University of Massachusetts at Boston, Dorchester, MASSACHUSETTS 02125

(S. Scheinberg): University of California Irvine, Irvine, California 92717 